arXiv:0809.2397 [hep-ph]

\title{
Selecting Finite Unified Theories with Current Data
}

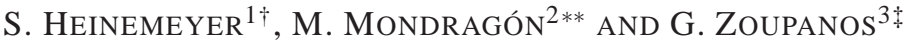 \\ ${ }^{1}$ Instituto de Fisica de Cantabria (CSIC-UC), Santander, Spain \\ ${ }^{2}$ Inst. de Fisica, Universidad Nacional Autónoma de México, México 01000, D.F., México \\ ${ }^{3}$ Physics Department, National Technical University of Athens, Athens, Greece
}

\begin{abstract}
Finite Unified Theories (FUTs) are $N=1$ supersymmetric Grand Unified Theories that can be made all-loop finite, leading to a severe reduction of the free parameters. We review the investigation of FUTs based on $S U(5)$ in the context of low-energy phenomenology observables. Using the restrictions from the top and bottom quark masses, it is possible to discriminate between different models. Including further low-energy constraints such as $B$ physics observables, the bound on the lightest Higgs boson mass and the cold dark matter density, we derive the predictions for the supersymmetric particle spectrum and the prospects for discoveries at the LHC.
\end{abstract}

* talk given at the SUSY 08, June 2008, Seoul, Korea

$\dagger$ email: Sven.Heinemeyer@cern.ch

** email: myriam@fisica.unam.mx

$\ddagger$ email: George.Zoupanos@cern.ch 


\title{
Selecting Finite Unified Theories with Current Data
}

\author{
S. Heinemeyer*, M. Mondragón ${ }^{\dagger}$ and G. Zoupanos** \\ *Instituto de Fisica de Cantabria (CSIC-UC), Santander, Spain \\ ${ }^{\dagger}$ Inst. de Fisica, Universidad Nacional Autónoma de México, México 01000, D.F., México \\ ${ }^{* *}$ Physics Department, National Technical University of Athens, Athens, Greece
}

\begin{abstract}
Finite Unified Theories (FUTs) are $N=1$ supersymmetric Grand Unified Theories that can be made all-loop finite, leading to a severe reduction of the free parameters. We review the investigation of FUTs based on $S U(5)$ in the context of low-energy phenomenology observables. Using the restrictions from the top and bottom quark masses, it is possible to discriminate between different models. Including further low-energy constraints such as $B$ physics observables, the bound on the lightest Higgs boson mass and the cold dark matter density, we derive the predictions for the supersymmetric particle spectrum and the prospects for discoveries at the LHC.
\end{abstract}

\section{INTRODUCTION}

Finite Unified Theories are $N=1$ supersymmetric GUTs which can be made finite even to all-loop orders, including the soft supersymmetry (SUSY) breaking sector, see Refs. [1, 2] for details and further references. The constructed finite unified $N=1$ supersymmetric GUTs predicted correctly from the dimensionless sector (GaugeYukawa unification), among others, the top quark mass [3]. Eventually, the predictive power has been extended to the Higgs sector and the SUSY spectrum. This, in turn, allows to make predictions for low-energy precision and astrophysical observables.

We review an exhaustive search $S U(5)$-based finite SUSY models, taking into account the restrictions resulting from the low-energy observables [4]1. Finally, the predictions of the "best" model (i.e. that is still allowed after taking the phenomenological restrictions into account) for the Higgs and SUSY searches at the LHC are reviewed [4].

\section{THE FINITE SU(5) MODELS}

From the classification of finite theories in Ref. [6], one can see that there exist only two candidate possibilities to construct $S U(5)$ GUTs with three generations. These possibilities require that the theory should contain as matter fields the chiral super-multiplets $\mathbf{5}, \overline{\mathbf{5}}, \mathbf{1 0}, \overline{\mathbf{5}}, \mathbf{2 4}$ with the multiplicities $(6,9,4,1,0)$ and $(4,7,3,0,1)$, respectively. Only the second one contains a 24-plet which can be used to provide the spontaneous symmetry break-

\footnotetext{
1 Analyses of other models based on low-energy precision observables
} can be found in Ref. [5] and references therein. ing (SB) of $S U(5)$ down to $S U(3) \times S U(2) \times U(1)$. Consequently, we concentrate on this class of models.

The particle content of the models we will study consists of the following super-multiplets: three $(\overline{\mathbf{5}}+\mathbf{1 0})$, needed for each of the three generations of quarks and leptons, four $(\overline{\mathbf{5}}+\mathbf{5})$ and one $\mathbf{2 4}$ considered as Higgs super-multiplets. When the gauge group of the finite GUT is broken the theory is no longer finite, and we will assume that we are left with the MSSM.

In the following we review two versions of the allorder finite model. The model of Ref. [3], which will be labeled $\mathbf{A}$, and a slight variation of this model (labeled B), which can also be obtained from the class of the models suggested in Ref. [7] (with a modification to suppress non-diagonal anomalous dimensions). The main difference between model $\mathbf{A}$ and model $\mathbf{B}$ is that two pairs of Higgs quintets and anti-quintets couple to the $\mathbf{2 4}$ in B, so that it is not necessary to mix them with $H_{4}$ and $\bar{H}_{4}$ in order to achieve the triplet-doublet splitting after the symmetry breaking of $S U(5)$ [2]. Thus, although the particle content is the same, the solutions to restrictions among the parameters differ, see Ref. [4] for details. FUTA depends on three free parameters, namely $m_{\overline{\mathbf{5}}} \equiv m_{\overline{\mathbf{5}}_{3}}, m_{\mathbf{1 0}} \equiv m_{\mathbf{1 0}_{3}}$ and $M$. Here $m_{\mathbf{N}}$ denote scalar mass parameters and $M$ the fermionic mass parameters at the GUT scale. FUTB is even more restricted and depends only on two free parameters, $m_{10} \equiv m_{10_{3}}$ and $M$ for the dimensionful sector.

\section{THE QUARK MASSES}

We start with the comparison of the predictions of the two models, FUTA and FUTB, for the top- and bottom quark masses with their experimental values. For the topquark mass we used the (older) experimental value for 

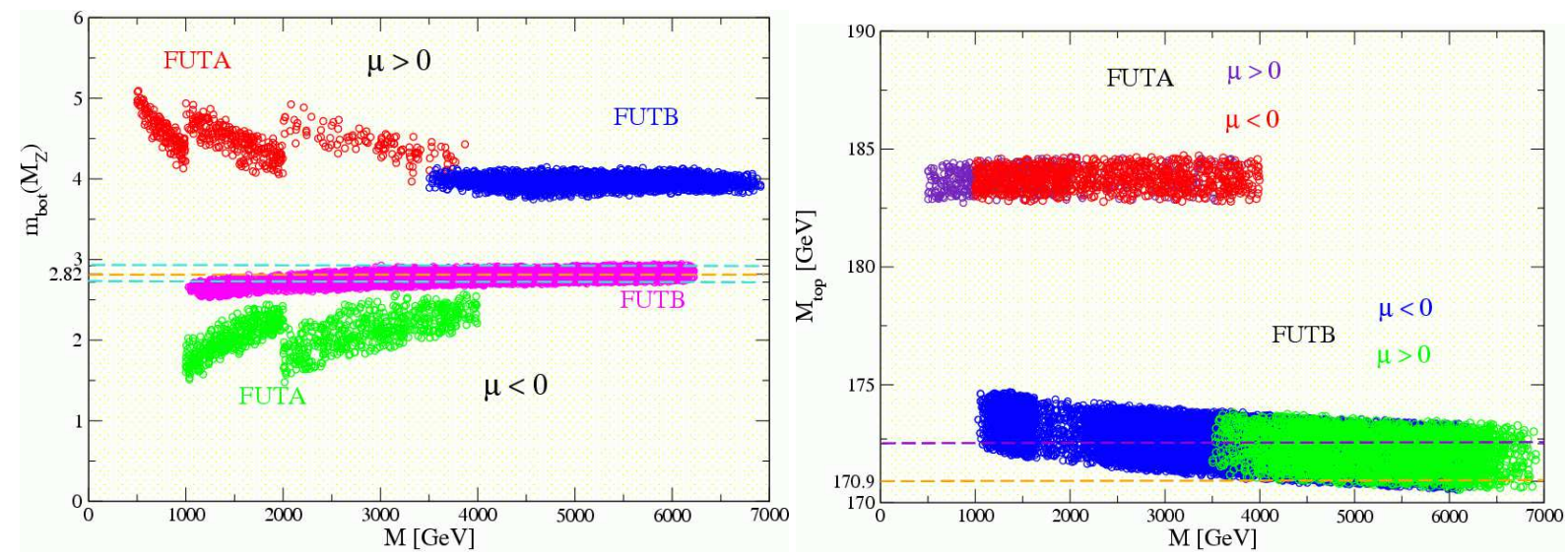

FIGURE 1. $\bar{m}_{b}\left(M_{Z}\right)$ as function of $M$ (left) and $m_{t}$ as function of $M$ (right) for models FUTA and FUTB, for $\mu<0$ and $\mu>0$.

the pole mass [8]

$$
m_{t}^{\exp }=170.9 \pm 1.8 \mathrm{GeV} .
$$

For the bottom-quark mass we use the value at the bottom-quark mass scale or at $M_{Z}$ [9]

$$
\bar{m}_{b}\left(M_{Z}\right)=2.82 \pm 0.07 \mathrm{GeV} .
$$

In Fig. 1 we present the predictions of the models concerning the bottom quark mass (left) and the top quark mass (right). The steps in the values for FUTA are due to the fact that fixed values of $M$ were taken, while the other parameters $m_{5}$ and $m_{10}$ were varied. However, this selected sampling of the parameter space is sufficient for us to draw our conclusions, see below. We present the predictions for $\bar{m}_{b}\left(M_{Z}\right)$, to avoid unnecessary errors coming from the running from $M_{Z}$ to the $m_{b}$ pole mass, which are not related to the predictions of the present models. The so-called $\Delta_{b}$ effects are taken into account, see Ref. [4] for more details. The bounds on the $\bar{m}_{b}\left(M_{Z}\right)$ and the top quark mass single out FUTB with $\mu<0$ as the most favorable solution. The favored parameter points have $\mu=\mathscr{O}(-2000 \mathrm{GeV})$ and $\tan \beta=\mathscr{O}(50)$.

Looking at the anomalous magnetic moment of the muon [10, 11, 12] (see Ref. [4] for a full list of references) it is obvious that $\mu<0$ is already challenged by the present data. However, a heavy SUSY spectrum as we have here (see below) with $\mu<0$ results in a $a_{\mu}^{\text {SUSY }}$ prediction very close to the SM result. Since the SM is not regarded as excluded by $(g-2)_{\mu}$, we continue with our analysis of FUTB with $\mu<0$.

\section{LHC PREDICTIONS}

We now turn to the predictions for the LHC based on model FUTB with $\mu<0$. Further experimental bounds applied are the ones of $\mathrm{BR}(b \rightarrow s \gamma)$ and $\mathrm{BR}\left(B_{s} \rightarrow\right.$ $\mu^{+} \mu^{-}$) (evaluated with Micromegas [13]), the LEP Higgs bounds [14] on the lightest Higgs boson mass (evaluated with FeynHiggs [15, 16, 17, 18]) and the abundance of cold dark matter (CDM) in the early universe (evaluated with Micromegas).

In the left plot of Fig. 2 we show the prediction of FUTB with $\mu<0$ for the lightest observable particle (LOSP) as function of $M$, that comply with the $B$ physics and Higgs constraints. The darker (red) points fulfill in addition the a loose CDM constraint (see Ref. [4]). The LOSP is either the light scalar $\tau$ or the second lightest neutralino (which is close in mass with the lightest chargino). One can see that the masses are outside the reach of the LHC and also the ILC. Neglecting the CDM constraint, even higher particle masses are allowed.

The right plot of Fig. 2 we show the prediction of FUTB with $\mu<0$ for the lighter scalar bottom, the lighter scalar top and the gluino as a function of $M$. The masses show a nearly linear dependence on $M$. Assuming a discovery reach of $\sim 2.5 \mathrm{TeV}$ yields a coverage up to $M \lesssim 2 \mathrm{TeV}$. This corresponds to the largest part of the CDM favored parameter space.

Finally in Fig. 3 we show the predictions for the heavy MSSM Higgs bosons as a function of the lightest Higgs boson mass. We have truncated the plot at about $M_{A}=$ $10 \mathrm{TeV}$. The parameter space allowed by $B$ physics extends up to $\sim 30 \mathrm{TeV}$. The values that comply with the CDM constraints are in a relatively light region of $M_{A}$ with $M_{A} \lesssim 4000 \mathrm{GeV}$. However, from 3 it follows that the LHC will observe only a light Higgs boson, whereas the heavy Higgs bosons remain outside the LHC reach, even for such large values of $|\mu|$ and $\tan \beta$ [19]. 

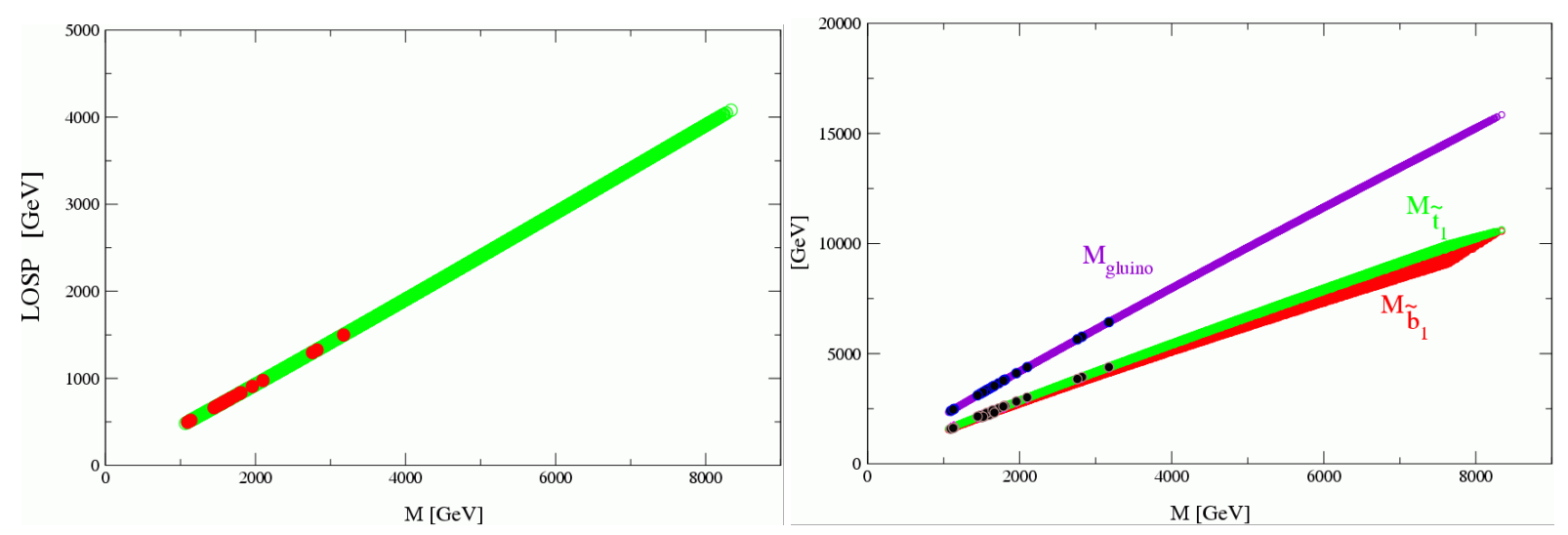

FIGURE 2. Predictions for SUSY masses of FUTB with $\mu<0$. The left (right) plot shows the lightest observable particle (the masses of the lighter scalar top, scalar bottom and gluino) as a function of $M$. The darker shaded (red/left, black/right) dots indicate the points in agreement with the CDM bound.

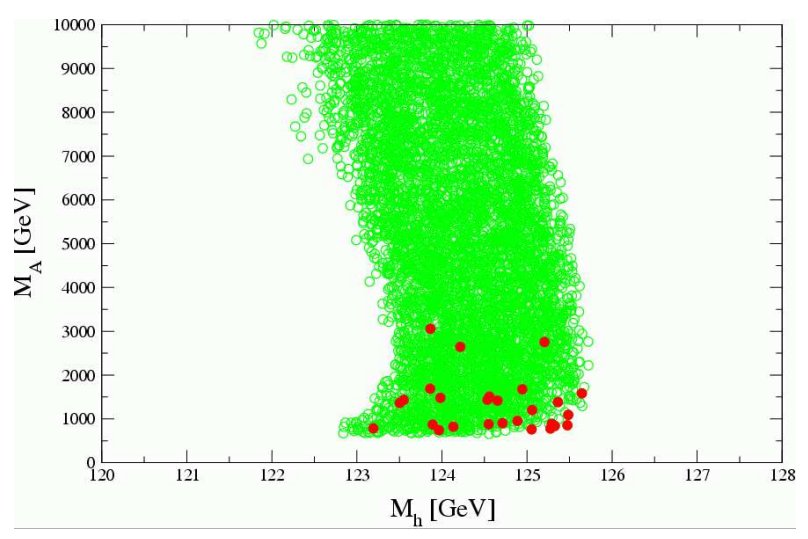

FIGURE 3. The heavy Higgs boson mass $M_{A}$ is shown as a function of the lightest Higgs mass $M_{h}$. The darker shaded (red) dots indicate the points in agreement with the CDM bound.

\section{ACKNOWLEDGMENTS}

Partially supported by the NTUA programme for basic research "K. Karatheodoris". Work supported in part by the EU's Marie-Curie Research Training Network under contract MRTN-CT-2006-035505 'Tools and Precision Calculations for Physics Discoveries at Colliders'. M.M. has been supported by the mexican projects PAPIIT IN115207 and Conacyt 51554-F.

\section{REFERENCES}

1. J. Kubo, M. Mondragón and G. Zoupanos, arXiv:hepph/9703289; T. Kobayashi, J. Kubo, M. Mondragón and G. Zoupanos, AIP Conf. Proc. 490 (1999) 279.

2. T. Kobayashi, J. Kubo, M. Mondragón and G. Zoupanos, Nucl. Phys. B 511 (1998) 45 [arXiv:hep-ph/9707425].

3. D. Kapetanakis, M. Mondragón and G. Zoupanos, Zeit.f.
Phys. C 60 (1993) 181; M. Mondragón and G. Zoupanos, Nucl. Phys. C 37 (1995) 98.

4. S. Heinemeyer, M. Mondragón and G. Zoupanos, JHEP 0807 (2008) 135 [arXiv:0712.3630 [hep-ph]].

5. O. Buchmueller et al., arXiv:0808.4128 [hep-ph];

S. Heinemeyer, arXiv:0809.2395 [hep-ph].

6. S. Hamidi, J. Patera and J. H. Schwarz, Phys. Lett. B 141 (1984) 349; S. Rajpoot and J. Taylor, Phys. Lett. B 147 (1984) 91; X. Jiang and X. Zhou, Commun. Theor. Phys. 5 (1986) 179.

7. L. Avdeev, D. Kazakov, I. Kondrashuk, Nucl. Phys. B 510 (1998) 289; D. Kazakov, Phys. Lett. B 449 (1999) 201.

8. Tevatron Electroweak Working Group, hep-ex/0703034.

9. W. Yao et al. [Particle Data Group Collaboration], J. Phys. G 33 (2006) 1.

10. G. Bennett et al. [The Muon g-2 Collaboration], Phys. Rev. D 73 (2006) 072003 [arXiv:hep-ex/0602035].

11. T. Moroi, Phys. Rev. D 53 (1996) 6565 [Erratum-ibid. D 56 (1997) 4424] [arXiv:hep-ph/9512396].

12. D. Stöckinger, J. Phys. G 34 (2007) R45 [arXiv:hep$\mathrm{ph} / 0609168]$.

13. G. Belanger, F. Boudjema, A. Pukhov and A. Semenov, Comput. Phys. Commun. 149 (2002) 103 [arXiv:hepph/0112278]; Comput. Phys. Commun. 174 (2006) 577 [arXiv:hep-ph/0405253].

14. LEP Higgs working group, Phys. Lett. B 565 (2003) 61 [arXiv:hep-ex/0306033]; Eur. Phys. J. C 47 (2006) 547 [arXiv:hep-ex/0602042].

15. S. Heinemeyer, W. Hollik and G. Weiglein, Comp. Phys. Commun. 124200076 [arXiv:hep-ph/9812320]. The code is accessible via http://www. feynhiggs. de.

16. S. Heinemeyer, W. Hollik and G. Weiglein, Eur. Phys. J. C 9 (1999) 343 [arXiv:hep-ph/9812472].

17. G. Degrassi, S. Heinemeyer, W. Hollik, P. Slavich, G. Weiglein, Eur. Phys. J. C 28 (2003) 133 [arXiv:hep$\mathrm{ph} / 0212020]$.

18. M. Frank et al., JHEP 0702 (2007) 047 [arXiv:hep$\mathrm{ph} / 0611326]$.

19. S. Gennai et al., Eur. Phys. J. C 52 (2007) 383 [arXiv:0704.0619 [hep-ph]]; M. Hashemi et al., arXiv:0804.1228 [hep-ph]; S. Heinemeyer, A. Nikitenko and G. Weiglein, arXiv:0809.2396 [hep-ph]; 